\title{
Contact with Fluoride-Releasing Restorative Materials Can Arrest Simulated Approximal Caries Lesion
}

\author{
Camila de Almeida Brandão Guglielmi, Ana Flávia Bissoto Calvo, Tamara Kerber Tedesco, \\ Fausto Medeiros Mendes, and Daniela Prócida Raggio \\ Department of Orthodontics and Pediatric Dentistry, University of São Paulo, Avenue Prof. Lineu Prestes, 2227 São Paulo, SP, Brazil \\ Correspondence should be addressed to Daniela Prócida Raggio; danielar@usp.br
}

Received 15 June 2015; Revised 3 August 2015; Accepted 9 August 2015

Academic Editor: Tae-Yub Kwon

Copyright (C) 2015 Camila de Almeida Brandão Guglielmi et al. This is an open access article distributed under the Creative Commons Attribution License, which permits unrestricted use, distribution, and reproduction in any medium, provided the original work is properly cited.

\begin{abstract}
Previous studies have suggested that the presence of white-spot lesion is very probable when adjacent surface is affected by cavitated lesions. This study evaluated the potential of different fluoride-releasing restorative materials in arresting enamel white-spot lesions in approximal surface in contact with them, in vitro (I) and in situ (II). White-spot lesions were formed in 240 primary enamel specimens via $\mathrm{pH}$-cycling. They were put in contact with cylindrical blocks of 6 materials $(n=20)$ : composite resin, 2 high-viscous glass ionomer cements (HVGIC), resin-modified GIC, resin-modified nanoionomer, and polyacid-modified resin. In both studies I and II, these settings were designed to simulate the contact point between the restoration and simulated approximal lesion. For study I, they were subjected to a new pH-cycling cariogenic challenge for 7 or 14 days $(n=10)$. For study II, a randomized doubleblind in situ design was conducted in two phases (7/14 days) to promote cariogenic challenge. At the end of both studies, specimens were collected for mineral analysis by cross-sectional microhardness. Higher mineral loss was observed for lesions in contact with resin $(p<0.001)$. HVGICs were the most efficient in preventing mineral loss, whereas other materials presented an intermediate behavior. It is concluded that fluoride-releasing materials can moderately reduce white-spot lesions progression, and HVGIC can arrest enamel lesion in approximal surface in contact with them.
\end{abstract}

\section{Introduction}

Particularly in pediatric dentistry, glass ionomer cements (GICs) have raised interest because of properties such as handling and fluoride release/uptake, conferring it an anticariogenic potential, in addition to its biocompatibility and thermal expansion coefficient similar to tooth [1]; however, poor mechanical properties of conventional GICs make them unsuitable for multiple-surfaces restorations [2].

Nowadays some variations have been proposed to overcome this issue. After observing that better performance might be achieved by enhancing the power/liquid ratio and consequently shortening the period of the acid-base setting reaction, high-viscous GICs (HVGICs) were developed and have become the material of choice to perform the Atraumatic Restorative Treatment (ART) [3]. Clinical studies demonstrated that the HVGIC is equally resistant when compared with amalgam for occlusal cavities in permanent teeth $[4,5]$ and also for occlusoproximal cavities in primary teeth [6].

Resin-modified glass ionomer cements (RMGICs) and polyacid-modified resin composites (PMR) have also been developed in an attempt to improve the wear resistance, moisture sensitivity, and esthetic characteristic of GIC, maintaining their fluoride-releasing capacity. Recently, a new generation of RMGIC containing nanoparticles was launched with the aim of improving aesthetics properties, as lower surface wear and staining resistance [7]. However, there is a lack of studies concerning mechanical properties and the anticariogenic effects of this material, even though it seems to present lower surface wear when compared to conventional and resin-modified GIC [8].

The benefits of using fluoride-releasing restorative materials to protect the restoration-tooth interface during a cariogenic challenge have been widely studied and positive results are usually reported [9]. These findings, together with 
those found in clinical studies [10-12], suggest that fluoridereleasing restorative materials could possibly extend their protective or therapeutic effect to the enamel of the tooth in approximal surface in contact with them. It would be interesting since approximal surface is considered an area of high plaque accumulation and the presence of white-spot lesion is very probable when the adjacent surface is affected by cavitated lesions [13]. Nevertheless, to the best of our knowledge, no previous studies have been conducted with these alternative restorative materials, especially RMGIC containing nanoparticles and encapsulated HVGIC. It seems that this last one presented an enhanced fluoride release due to a predetermined powder/liquid proportion [14], which can result in a greater benefit to arrest the initial caries lesion in approximal surface.

Thus, our purpose was to evaluate the potential of different fluoride-releasing restorative materials in arresting enamel white-spot lesions in approximal surface in contact with them, under in vitro and in situ cariogenic challenges.

\section{Materials and Methods}

2.1. Specimen Preparation and Caries Induction. This study was approved by the ethical board of the University of São Paulo (\#186/2009) and teeth were obtained from the Human Tooth Bank of the University. Two hundred and forty blocks $(5 \times 5 \times 3 \mathrm{~mm})$ were obtained from the buccal surface of primary canines. Teeth were free of apparent enamel defects, macroscopic cracks, abrasions, and staining, assessed at $2 \mathrm{x}$ magnification with a stereomicroscope (Stereo Discovery V20, Zeiss, Göttingen, NI, Germany). They were cut using a double-face diamond disc (KG Sorensen, São Paulo, SP, Brazil) in a slow-speed handpiece under water irrigation. After that, all slabs surfaces were cleaned with rotating brushes/abrasive paste, washed with deionized water, and maintained in relative humidity. To maintain the convexity of the surface, no other treatment was performed.

The specimens were made completely impermeable with two coats of acid-resistant nail varnish, except for an area of $3 \times 2 \mathrm{~mm}$ on the center of enamel surface. All of them were submitted to the $\mathrm{pH}$-cycling procedure to create artificial incipient caries lesions. The demineralizing solution contained $2.2 \mathrm{mM} \mathrm{CaCl}, 2.2 \mathrm{mM} \mathrm{NaH} \mathrm{PO}_{4}$, and $50 \mathrm{mM}$ acetic acid adjusted to $\mathrm{pH}$ 4.6. The remineralizing solution contained $1.5 \mathrm{mM} \mathrm{CaCl}_{2}, 0.9 \mathrm{mM} \mathrm{NaH}_{2} \mathrm{PO}_{4}$, and $0.15 \mathrm{M} \mathrm{KCl}$ adjusted to $\mathrm{pH}$ 7.0. Each specimen was cycled in $2 \mathrm{~mL}$ for 8 hours in the demineralizing solution and 16 hours in the remineralizing solution at room temperature and without agitation, during 10 days. A new solution was used every cycle $[15,16]$.

2.2. Restorative Material Preparation. Cylindrical samples $(4 \mathrm{~mm}$ in diameter $\times 3 \mathrm{~mm})$ were prepared using six restorative materials (composite resin, HVGIC, encapsulated HVGIC, RMGIC, nanoparticle RMGIC, and PMR) using a circular matrix according to manufacturers' instructions (Table 1). Photoactivated materials were accommodated into the matrix in one increment and light-cured using a halogenbased light-curing unit. Self-cured materials were placed in a single bulk with an insertion spatula, except for encapsulated HVGIC and PMR because they are capsulated materials and are delivered by syringe. After curing, they were removed from matrix by pressure.

All samples were stored in liquid petroleum jelly for a period of 24 hours [17]. After this period, they were cleaned with gauze and put in contact with the convex surface of enamel slab containing white-spot lesion and fixed with orthodontic band. This setting was designed to simulate the contact point between an occlusoproximal restoration and the adjacent tooth (Figure 1).

2.3. In Vitro Cariogenic Challenge. Half of the specimens $(n=120)$ were submitted to an in vitro cariogenic challenge for $7(n=60)$ or $14(n=60)$ days, being immersed on demineralizing and remineralizing solutions with the same composition used for caries lesion induction. Each specimen (enamel + material block) was cycled in $3 \mathrm{~mL}$ for 8 hours in the demineralizing solution and 16 hours in the remineralizing solution, which had the $\mathrm{pH}$ adjusted for 4.5.

2.4. In Situ Cariogenic Challenge. The other half of the specimens $(n=120)$ were submitted to an in situ cariogenic challenge conducted in two phases of $7(n=60)$ or $14(n=60)$ days, with a period of 7-day washout between them. For this purpose, specimens (enamel block + material block) were inserted in acrylic custom-made palatal devices containing 6 cavities $(6 \times 5 \times 5 \mathrm{~mm}), 3$ on the left and 3 on the right side. To better fit the chambers, specimens' dimensions were reduced to $4 \times 4 \times 2 \mathrm{~mm}$. The chambers were open both to the palatal and to the buccal surfaces and into each of them one slab was fixed with wax. A plastic mesh was fixed with acrylic resin over the chambers, leaving a $1 \mathrm{~mm}$ space from the specimen to allow biofilm accumulation and protect it from mechanical disturbance.

To avoid any possible benefit caused by its positions, distribution of specimen was randomly determined according to a computer-generated randomization list for the first appliance mounted. For the following appliances, the position of samples was evenly rotated so that each material occupied the 6 positions.

Ten volunteers from São Paulo (water fluoride level $0.7 \mathrm{mg} \mathrm{L}^{-1}$ ) were invited to take part in the study based on the following inclusion and exclusion criteria: age between 20 and 40 years; having at least 20 teeth; absence of active caries lesions; no use of fixed or removable orthodontic device; no use of any antibiotics within the 2 months prior to study beginning. They also had to agree with the study terms and be willing to abstain from oral hygiene products except those provided. The study was designed in two phases of $7(n=$ $60)$ and $14(n=60)$ days, with a period of 7-day washout between them. Thus, half of the volunteers initiated by the 7days period and the other half initiated by the 14-days period.

Volunteers were instructed to wear their appliance 24 hours per day and during all the experimental period they brushed their teeth with a fluoridated dentifrice (Colgate 
TABLE 1: Description of materials tests in the study.

\begin{tabular}{|c|c|c|c|}
\hline Restorative material & Name & Composition & $\begin{array}{l}\text { Insertion and } \\
\text { polymerization method }\end{array}$ \\
\hline $\begin{array}{l}\text { Nanocomposite } \\
\text { composite resin }\end{array}$ & $\begin{array}{l}\text { Z350 (3M } \\
\text { ESPE, St. } \\
\text { Paul, MN, } \\
\text { USA) }\end{array}$ & $\begin{array}{l}\text { Silane treated ceramic } \\
\text { Silane treated silica } \\
\text { Diurethane dimethacrylate (udma) } \\
\text { Bisphenol a polyethylene glycol diether dimethacrylate } \\
\text { Bisphenol a diglycidyl ether dimethacrylate (bisgma) } \\
\text { Silane treated zirconia } \\
\text { Polyethylene glycol dimethacrylate } \\
\text { Triethylene glycol dimethacrylate (tegdma) } \\
\text { 2,6-Di-tert-butyl-p-cresol }\end{array}$ & $\begin{array}{l}2 \text { increments }(2 \mathrm{~mm}) / 40 \mathrm{~s} \\
\text { photoactivation }\end{array}$ \\
\hline $\begin{array}{l}\text { High-viscous } \\
\text { CIV }\end{array}$ & $\begin{array}{l}\text { Ketac Molar } \\
\text { (3M ESPE, St. } \\
\text { Paul, MN, } \\
\text { USA) }\end{array}$ & $\begin{array}{l}\text { Powder: } \\
\text { Glass powder } \\
\text { Polyacrylic acid } \\
\text { Liquid: } \\
\text { Water } \\
\text { Copolymer of acrylic acid-maleic acid } \\
\text { Tartaric acid }\end{array}$ & $\begin{array}{l}\text { Insertion spatula; finger } \\
\text { press technique with solid } \\
\text { petroleum jelly }\end{array}$ \\
\hline $\begin{array}{l}\text { Encapsulated } \\
\text { high-viscous } \\
\text { GIC }\end{array}$ & $\begin{array}{l}\text { Riva Self } \\
\text { Cure (SDI, } \\
\text { Bayswater; } \\
\text { VIC, } \\
\text { Australia) }\end{array}$ & $\begin{array}{l}\text { Powder: polyacrylic acid, aluminosilicate glass Liquid: } \\
\text { polyacrylic acid, tartaric acid }\end{array}$ & $\begin{array}{l}\text { Delivery syringe; finger } \\
\text { press technique with solid } \\
\text { petroleum jelly }\end{array}$ \\
\hline $\begin{array}{l}\text { Resin-modified } \\
\text { GIC }\end{array}$ & $\begin{array}{l}\text { Vitremer ( } 3 \mathrm{M} \\
\text { ESPE, St. } \\
\text { Paul, MN, } \\
\text { USA) }\end{array}$ & $\begin{array}{l}\text { Powder: } \\
\text { Silane treated glass } \\
\text { Potassium persulfate } \\
\text { Liquid: } \\
\text { Copolymer of acrylic and itaconic acids } \\
\text { Water } \\
\text { 2-Hydroxyethyl methacrylate (hema) } \\
\text { Diphenyliodonium hexafluorophosphate }\end{array}$ & $\begin{array}{l}\text { Insertion spatula; finger } \\
\text { press technique with solid } \\
\text { petroleum jelly }\end{array}$ \\
\hline $\begin{array}{l}\text { Resin-modified } \\
\text { GIC with nanoparticles }\end{array}$ & $\begin{array}{l}\text { Ketac Nano } \\
\text { (3M ESPE, St. } \\
\text { Paul, MN, } \\
\text { USA) }\end{array}$ & $\begin{array}{l}\text { Silane treated glass } \\
\text { Silane treated zirconia } \\
\text { Polyethylene glycol dimethacrylate (pegdma) } \\
\text { Silane treated silica } \\
\text { 2-Hydroxyethyl methacrylate (hema) } \\
\text { Glass powder } \\
\text { Bisphenol a diglycidyl ether dimethacrylate (bisgma) } \\
\text { Triethylene glycol dimethacrylate (tegdma) }\end{array}$ & $\begin{array}{l}2 \text { increments }(2 \mathrm{~mm}) / 20 \mathrm{~s} \\
\text { photoactivation }\end{array}$ \\
\hline Polyacid-modified resin & $\begin{array}{l}\text { Dyract Extra } \\
\text { (Dentsply, } \\
\text { Konstanz } \\
\text { BW, } \\
\text { Germany) }\end{array}$ & $\begin{array}{l}\text { Ethoxylated bisphenol-A-dimethacrylate, urethane resin, } \\
\text { triethylene glycol dimethacrylate (TEGDMA), and } \\
\text { trimethylolpropane trimethacrylate } \\
\text { (TMPTMA) } \\
\text { and strontium fluoride glass }\end{array}$ & $\begin{array}{l}2 \text { increments }(2 \mathrm{~mm}) / 20 \mathrm{~s} \\
\text { photoactivation }\end{array}$ \\
\hline
\end{tabular}

Total 12-1450 mg F/g, Colgate-Palmolive, São Paulo, SP, Brazil). The cariogenic challenge was provided by dripping one drop of $20 \%$ sucrose solution over the mesh onto each specimen, 8 times per day at predetermined times. Before reinsertion in the mouth, the appliance was kept on their plastic recipient for $5 \mathrm{~min}$ to allow sucrose solution diffusion. The use of dentifrice was performed at least 3 times a day, after meal-times, and when volunteers were habituated to perform oral hygiene. The appliances were extraorally brushed only on the palatal contact surface. When not in the oral cavity (meals and oral hygiene), the appliances were kept moist in their plastic recipients.

After the intraoral experimental phase, the appliances were collected and specimens were removed. The enamel blocks were separated from the cylindrical material blocks and then brushed gently with a very soft toothbrush to remove biofilm.

2.5. Cross-Section Microhardness Analysis (CSMH). Enamel blocks were longitudinally sectioned with double-face diamond discs (KG Sorensen, Cotia, SP, Brazil) through the center of window left for caries induction. One half of each sample was embedded in acrylic resin. The surfaces of the samples were planned with an automatic grinding/polishing machine and $\mathrm{SiC}$ discs of \#400, \#600, \#1200, and \#1400 grit under running water for $60 \mathrm{~s}$ and then polished with diamond paste ( 1 and $0.25 \mu \mathrm{m}$ ). Cross-section microhardness measurements of the adjacent enamel were taken using a Knoop 


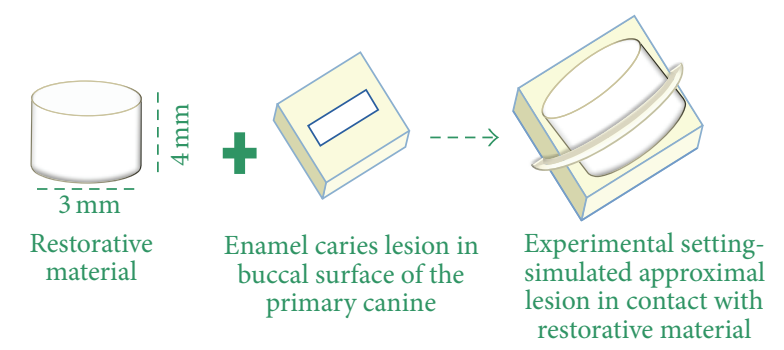

FIGURE 1: Experimental setting of the simulated approximal caries lesion in contact with restorative material.

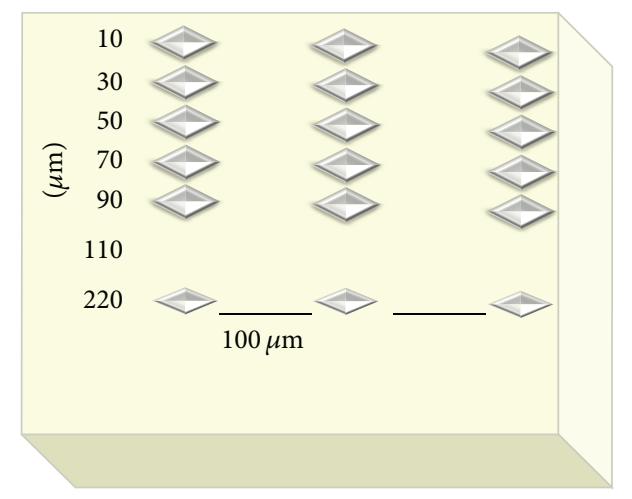

FIGURE 2: Diagram of microhardness measurements performed on each tooth specimen.

indenter attached to a microhardness tester (Shimadzu Micro Hardness Tester HMV-2; Shimadzu Corporation, Kyoto, KYO, Japan). Three rows of 7 indentations each were made, one in the central region of the dental enamel exposed and the other two at a $100 \mu \mathrm{m}$ distance to both sides of the central row using a 25-gram load for 15 seconds. The indentations were made at $10,30,50,70,90,110$, and $220 \mu \mathrm{m}$ from the outer enamel surface (Figure 2). The mean values of the 3 rows measuring points at each distance from the surface were averaged. To obtain values from sound enamel (baseline), 5 indentations were also performed over the area surrounding the window left for caries development and the average was used to represent this value.

2.6. Statistical Analyses. Values from CSMH at the different distances were used to construct a graphic (CSMH value versus distance form enamel surface) and the value corresponding to the area below the curve was considered. With regard to reference values from sound enamel (baseline), the average among the 5 indentations performed was used to construct the graph, using the same distances as those performed for the experimental part. Subtracting the experimental value from the baseline value, the surface microhardness value change was obtained and used to perform statically analysis.

Normal distribution of data and equality of variances were confirmed using Anderson-Darling and Levene tests, respectively. Subsequently, ANOVA and Tukey's test were carried out for statistical comparisons amongst restorative

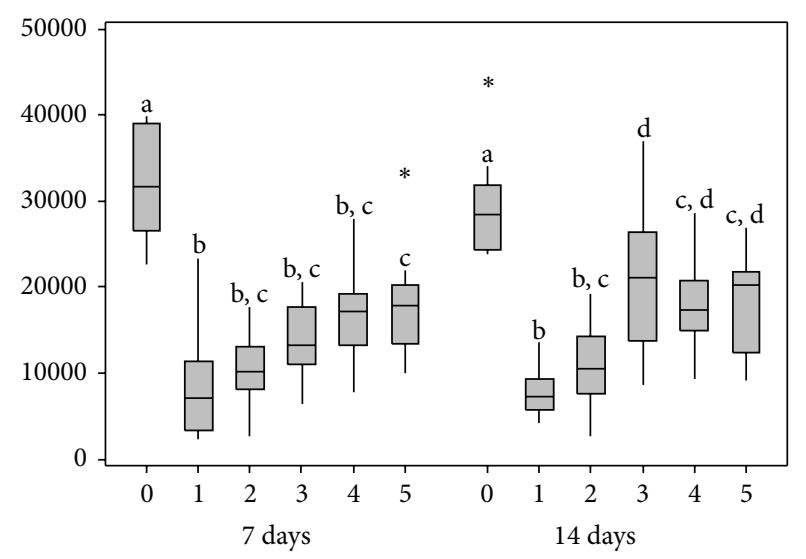

FIGURE 3: Cross-sectional microhardness results for in vitro study. Different letters show statistically significant differences $(p \leq 0.05)$. 0 = composite resin; 1 = HVGIC; 2 = encapsulated HVGIC; $3=$ RMGIC; 4 = nanoparticle RMGIC; 5 = PMR.

materials after in vitro challenge ( $\alpha=5 \%)$. For in situ study, multilevel analysis (volunteer and specimen) was performed also at a significance level of $5 \%$. All analyses were conducted using MLwin 2.10 Software (Centro for Multilevel Modeling, University of Bristol, Bristol, UK).

\section{Results}

3.1. In Vitro Study. Considering both periods of cariogenic challenge, specimens in contact with composite resin presented the highest mineral loss when comparing to all other materials. For the 7-day period, the HVGIC demonstrated greater capacity for inhibiting artificial caries lesions adjacent to restorations when compared to PMR. For the 14day period, the inhibitory capacity of the artificial caries lesion from HVGIC was also superior when compared to nanoparticle RMGIC and RMGIC. Results from the in vitro study are demonstrated in Figure 3.

3.2. In Situ Study. Within the 7-day period of cariogenic challenge, the HVGICs presented the best performance and the composite resin the worst performance in protecting the adjacent caries lesions, whereas the other materials presented an intermediate behavior. For the 14-day period of cariogenic challenge, materials presented a similar behavior, except that nanoparticle RMGIC was as inefficient as composite resin.

Furthermore, the behavior of composite resin was similar in both methods of cariogenic challenge. There was no statistically significant difference between the two HVGICs (HVGIC and encapsulated HVGIC). Results from the in situ study are shown in Figure 4.

\section{Discussion}

This study was designed to investigate the effects of different fluoride-releasing restorative materials in arresting initial enamel caries lesion in approximal surface in contact with them, under in vitro and in situ conditions. Thus, only 


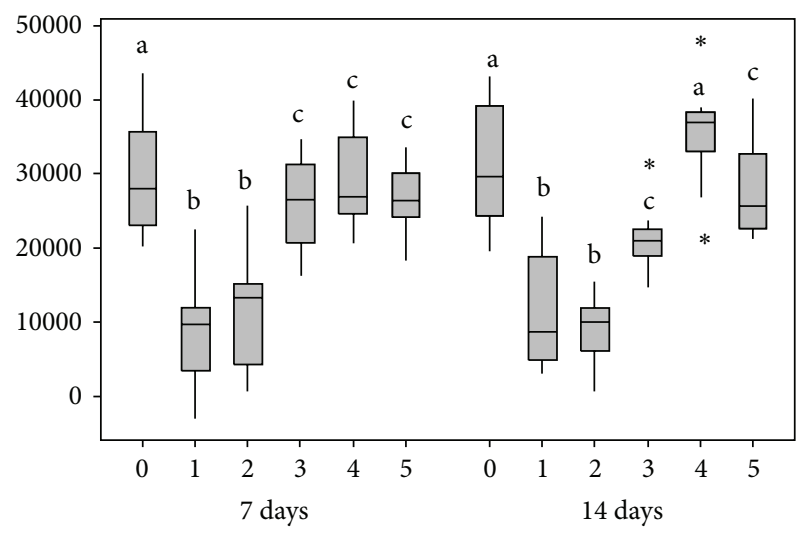

FIgURE 4: Cross-sectional microhardness results for in situ study. Different letters show statistically significant differences $(p \leq 0.05)$. 0 = composite resin; 1 = HVGIC; 2 = encapsulated HVGIC; $3=$ RMGIC; 4 = nanoparticle RMGIC; 5 = PMR.

materials indicated to perform occlusoproximal restorations were used in the study.

In general, the results of the study show that all fluoridereleasing materials are capable of arresting, in a greater or lesser extent, the progression of approximal initial caries lesion in contact with them. HVGICs presented the best performance, whereas other materials presented an intermediate performance between the pure GICs and the composite resin.

According to Wiegand et al. [18], fluoride release characteristics depend on the matrices, fillers, and fluoride content as well as the setting mechanisms and environmental conditions of the restoratives. However, it is commonly assumed that materials presenting higher fluoride release levels are those with poor mechanical properties [19]. In our study, the best anticariogenic potential verified for HVGIC can be explained by its chemical nature. An initial high release from GIC over the first $24 \mathrm{~h}$ occurs due to the burst of fluoride resulting from the glass particles when reacting with the polyalkenoate acid during the setting reaction [20]. After that, fluoride is released by both a short-term reaction involving a rapid dissolution from the outer surface into solution and a gradual reaction resulting by the sustained diffusion of ions through the bulk cement.

This finding corroborates a previous study that HVGIC resulted in a superior capacity to protect incipient lesions in approximal surface in contact with them when compared to other materials [21], being that the reservoir property can turn this even more effective. The permeability of the materials is a major factor in the mechanism of fluoride uptake, once they could absorb the ions deep into its bulk. The more permeable the material, the more likely its ability to absorb and release fluoride and composites are known to have relatively low water permeability, whereas the HVGIC and RMGIC have been shown to have greater sorption values [22]. It is also suggested that, in general, materials with higher initial fluoride release have higher recharge capability, even though original values are hardly reached again [23].

Two HVGICs were included in the study, since one of them is presented in the conventional powder/liquid form and the other is disposed in capsules. It has been suggested that encapsulated GICs can present an enhanced fluoride release once it has a predetermined powder/liquid proportion which facilitates handling and avoids operator and environmental induced variability [14], optimizing its performance. In our study, however, no difference was observed between the performances of these two types of HVGIC.

On the other hand, RMGIC usually demonstrates a comparable potential for releasing fluoride as conventional cements, but they may be affected not only by the formation of complex fluoride compounds and their interaction with polyacrylic acid, but also by the type and amount of resin used for the photochemical reaction [24]. Probably, it can be an explanation for the similar results verified for both RMGIC and RMGIC with nanoparticles in the present study, except for the 14-day period of in situ cariogenic challenge.

In fact, nanoparticle RMGIC seems to be less hydrophilic than conventional RMGIC [19]. Contrary to RMGIC, the first phase of setting for PMR is the light-activated polymerization, which means that it behaves initially as composites resins. In this way, after curing and before the contact with water, the fluoride is not free but bound in the filler particles, which are enclosed in the polymerized matrix. After the succeeding water sorption, the acid-base reaction takes place but the continuing fluoride release over time seems to be capable of protecting enamel in contact with these materials under cariogenic challenge [25].

This new generation of RMGIC, the so-called nanoionomers, presents as paste/paste system and is based on bonded nanofiller technology, aiming to promote an improved wear resistance. In fact, some studies have demonstrated lower roughness indices when compared to conventional GIC and RMGIC [8]; however, poor results have been achieved with regard to marginal adaptation and staining when compared to other materials such as conventional RMGIC and composite resin in a clinical trial [26]. Nevertheless, considering its anticariogenic effect, previous study observed better capacity to protect enamel around restorations under a cariogenic challenge than composite resin [27], as observed in this study.

The duration of cariogenic challenge, in general, exerted little influence over the performance of each material, mainly for pure GICs. Probably, the fluoride release for these materials drops moderately 7 days after manipulation. Only for nanoparticle RMGIC the results were similar to those for composite resin after 14 days of in situ cariogenic challenge, which is probably associated with its poor capacity to act as a fluoride reservoir [19].

Some studies had already investigated the potential of fluoride-releasing restorative materials to protect the adjacent enamel in approximal contact with them; however this effect is usually tested on sound surfaces [27-29]. We preferred to test the therapeutic effect of fluoride on incipient caries lesion once this condition is most likely to occur clinically, especially if a cavitated lesion is present on the adjacent tooth [13]. Also, unlikely to our study, most of them are not performed with primary teeth, the major indication for fluoride-releasing restorative material. In this way, the design of our study as well as the experimental setting seems to be effective and innovator to test our hypothesis. The enamel specimens were obtained from the buccal surface of deciduous canines 
and its curved shape was purposely preserved to simulate adequately the contact point against the cylindrical material block. Conversely the method chosen to develop the enamel caries lesions in both studies (in vitro and in situ), $\mathrm{pH}$ cycling, was previously demonstrated to be suitable when investigating the role of fluoride in the de-/remineralization process [30,31].

Even though most studies have tested the fluoride release potential of the restorative materials in deionized water or artificial saliva, the aim of this study was to test it in similar conditions to those found during the cariogenic challenge in mouth. It is known that acidic conditions can potentiate both the fluoride release from restoratives and the hydroxyapatite incorporation by enamel $[19,32]$, which may have favored their performance. However, the $\mathrm{pH}$ drop is also the circumstance in which there is the greatest need of the protection promoted by fluoride. Studies also usually demonstrate that fluoride leached from different fluoridated restorative materials may last for a long period especially for pure GIC; however, after a higher initial release, it drops to lower levels. As the aim of this study was to investigate the therapeutic potential of those materials, the short-term results (7 or 14 days) are more important than long-term results. Moreover, the use of in situ design assesses some characteristics that can influence the benefit to use fluoridereleasing restorative materials. The absence of other sources of fluoride from in vitro studies, as fluoridate toothpaste, can overestimate the effect of fluoride-releasing restorative materials in arresting initial caries lesion, being the other sources of fluoride sometimes enough to prevent or to arrest caries lesions. Moreover, the presence of biofilm and saliva can also interfere in the effect of these restorative materials.

Many factors must be taken into consideration when selecting a restorative material for primary dentition, including the ease of handling, their physical, chemical, and biological properties, and the possible longevity of the restorations. Although RMGIC, PMR, and composites are generally found to have a superior longevity when comparing to conventional GIC, HVGICs have demonstrated similar longevity to amalgam when performing occlusal restorations permanently [4]. According to Qvist et al. [2], restorations involving the approximal surface are those with the lowest survival rates in deciduous teeth. It should be emphasized, however, that there is no evidence for indicating some restorative material instead of others when considering occlusoproximal restorations on primary teeth [6].

\section{Conclusions}

Fluoride-releasing materials can moderately reduce whitespot lesions progression in surface in contact with them when compared to composite resin. However, HVGIC represents the best choice to arrest initial enamel lesion in approximal adjacent surfaces.

\section{Conflict of Interests}

The authors declare that there is no conflict of interests regarding the publication of this paper.

\section{Acknowledgments}

The authors would like to thank FAPESP (no. 2010/161207) and CAPES for financial support. Daniela Prócida Raggio and Fausto Medeiros Mendes receive Research Productivity Scholarship, CNPq.

\section{References}

[1] G. J. Mount, "Glass ionomers: a review of their current status," Operative Dentistry, vol. 24, no. 2, pp. 115-124, 1999.

[2] V. Qvist, A. Poulsen, P. T. Teglers, and I. A. Mjör, “The longevity of different restorations in primary teeth," International Journal of Paediatric Dentistry, vol. 20, no. 1, pp. 1-7, 2010.

[3] M. A. van 't Hof, J. E. Frencken, W. H. van Palenstein Helderman, and C. J. Holmgren, "The atraumatic restorative treatment (ART) approach for managing dental caries: a meta-analysis," International Dental Journal, vol. 56, no. 6, pp. 345-351, 2006.

[4] J. E. Frencken, M. A. Van 't Hof, W. E. Van Amerongen, and C. J. Holmgren, "Effectiveness of single-surface ART restorations in the permanent dentition: a meta-analysis," Journal of Dental Research, vol. 83, no. 2, pp. 120-123, 2004.

[5] S. Mickenautsch and V. Yengopal, "Failure rate of high-viscosity GIC based ART compared with that of conventional amalgam restorations-evidence from an update of a systematic review," SADJ, vol. 67, no. 7, pp. 329-331, 2012.

[6] D. P. Raggio, D. Hesse, T. L. Lenzi, C. Guglielmi, and M. M. Braga, "Is Atraumatic restorative treatment an option for restoring occlusoproximal caries lesions in primary teeth? A systematic review and meta-analysis," International Journal of Paediatric Dentistry, vol. 23, no. 6, pp. 435-443, 2013.

[7] S. B. Mitra, J. D. Oxman, A. Falsafi, and T. T. Ton, "Fluoride release and recharge behavior of a nano-filled resin-modified glass ionomer compared with that of other fluoride releasing materials," American Journal of Dentistry, vol. 24, no. 6, pp. 372378, 2011.

[8] S. B. P. de Fúcio, A. B. de Paula, F. G. de Carvalho, V. P. Feitosa, G. M. B. Ambrosano, and R. M. Puppin-Rontani, "Biomechanical degradation of the nano-filled resin-modified glass-ionomer surface," The American Journal of Dentistry, vol. 25, no. 6, pp. 315-320, 2012.

[9] S. Mickenautsch, V. Yengopal, and A. Banerjee, "Atraumatic restorative treatment versus amalgam restoration longevity: a systematic review," Clinical Oral Investigations, vol. 14, no. 3, pp. 233-240, 2010.

[10] V. Qvist, L. Laurberg, A. Poulsen, and P. T. Teglers, "Eight-year study on conventional glass ionomer and amalgam restorations in primary teeth," Acta Odontologica Scandinavica, vol. 62, no. 1, pp. 37-45, 2004.

[11] V. Qvist, L. Laurberg, A. Poulsen, and P. T. Teglers, "Class II restorations in primary teeth: 7-Year study on three resinmodified glass ionomer cements and a compomer," European Journal of Oral Sciences, vol. 112, no. 2, pp. 188-196, 2004.

[12] V. Qvist, E. Manscher, and P. T. Teglers, "Resin-modified and conventional glass ionomer restorations in primary teeth: 8year results," Journal of Dentistry, vol. 32, no. 4, pp. 285-294, 2004.

[13] M. G. Cagetti, G. Campus, S. Sale, F. Cocco, L. Strohmenger, and P. Lingström, "Association between interdental plaque acidogenicity and caries risk at surface level: a cross sectional study in primary dentition," International Journal of Paediatric Dentistry, vol. 21, no. 2, pp. 119-125, 2011. 
[14] R. P. Sousa, I. C. J. Zanin, J. P. M. Lima et al., "In situ effects of restorative materials on dental biofilm and enamel demineralisation," Journal of Dentistry, vol. 37, no. 1, pp. 44-51, 2009.

[15] F. M. Mendes and J. Nicolau, "Utilization of laser fluorescence to monitor caries lesions development in primary teeth," Journal of Dentistry for Children, vol. 71, no. 2, pp. 139-142, 2004.

[16] J. M. ten Cate and P. P. E. Duijsters, "Alternating demineralization and remineralization of artificial enamel lesions," Caries Research, vol. 16, no. 3, pp. 201-210, 1982.

[17] D. P. Raggio, C. C. Bonifácio, M. Bönecker, J. C. P. Imparato, A. J. de Gee, and W. E. van Amerongen, "Effect of insertion method on knoop hardness of high viscous glass ionomer cements," Brazilian Dental Journal, vol. 21, no. 5, pp. 439-445, 2010.

[18] A. Wiegand, W. Buchalla, and T. Attin, "Review on fluoridereleasing restorative materials-fluoride release and uptake characteristics, antibacterial activity and influence on caries formation," Dental Materials, vol. 23, no. 3, pp. 343-362, 2007.

[19] J. L. Moreau and H. H. K. Xu, "Fluoride releasing restorative materials: effects of $\mathrm{pH}$ on mechanical properties and ion release," Dental Materials, vol. 26, no. 11, pp. e227-e235, 2010.

[20] J. W. Nicholson, "Chemistry of glass-ionomer cements: a review," Biomaterials, vol. 19, no. 6, pp. 485-494, 1998.

[21] C. B. Marinelli, K. J. Donly, J. S. Wefel, J. R. Jakobsen, and G. E. Denehy, "An in vitro comparison of three fluoride regimens on enamel remineralization," Caries Research, vol. 31, no. 6, pp. 418-422, 1997.

[22] N. Attar and A. Önen, "Fluoride release and uptake characteristics of aesthetic restorative materials," Journal of Oral Rehabilitation, vol. 29, no. 8, pp. 791-798, 2002.

[23] N. Attar and M. D. Turgut, "Fluoride release and uptake capacities of fluoride-releasing restorative materials," Operative Dentistry, vol. 28, no. 4, pp. 395-402, 2003.

[24] G. J. Mount, C. Patel, and O. F. Makinson, "Resin modified glassionomers: strength, cure depth and translucency," Australian Dental Journal, vol. 47, no. 4, pp. 339-343, 2002.

[25] Á. M. Lennon, A. Wiegand, W. Buchalla, and T. Attin, "Approximal caries development in surfaces in contact with fluoridereleasing and non-fluoride-releasing restorative materials: an in situ study," European Journal of Oral Sciences, vol. 115, no. 6, pp. 497-501, 2007.

[26] J. Perdigão, M. Dutra-Corrêa, S. H. C. Saraceni, M. T. Ciaramicoli, and V. H. Kiyan, "Randomized clinical trial of two resinmodified glass ionomer materials: 1-year results," Operative Dentistry, vol. 37, no. 6, pp. 591-601, 2012.

[27] C. F. C. Salas, C. A. B. Guglielmi, D. P. Raggio, and F. M. Mendes, "Mineral loss on adjacent enamel glass ionomer cements restorations after cariogenic and erosive challenges," Archives of Oral Biology, vol. 56, no. 10, pp. 1014-1019, 2011.

[28] K.-T. Jang, F. Garcia-Godoy, K. J. Donly, and A. Segura, "Remineralizing effects of glass ionomer restorations on adjacent interproximal caries," Journal of Dentistry for Children, vol. 68, no. 2, pp. 125-128, 2001.

[29] L. Papagiannoulis, A. Kakaboura, and G. Eliades, "In vivo vs in vitro anticariogenic behavior of glass-ionomer and resin composite restorative materials," Dental Materials, vol. 18, no. 8, pp. 561-569, 2002.

[30] A. C. Magalhães, B. M. Moron, L. P. Comar, A. Wiegand, W. Buchalla, and M. A. R. Buzalaf, "Comparison of cross-sectional hardness and transverse microradiography of artificial carious enamel lesions induced by different demineralising solutions and gels," Caries Research, vol. 43, no. 6, pp. 474-483, 2009.
[31] A. E. De Mello Vieira, A. C. Botazzo Delbem, K. T. Sassaki, E. Rodrigues, J. A. Cury, and R. F. Cunha, "Fluoride dose response in $\mathrm{pH}$-cycling models using bovine enamel," Caries Research, vol. 39, no. 6, pp. 514-520, 2005.

[32] J. A. Cury and L. M. A. Tenuta, "Enamel remineralization: controlling the caries disease or treating early caries lesions?" Brazilian Oral Research, vol. 23, no. 1, pp. 23-30, 2009. 

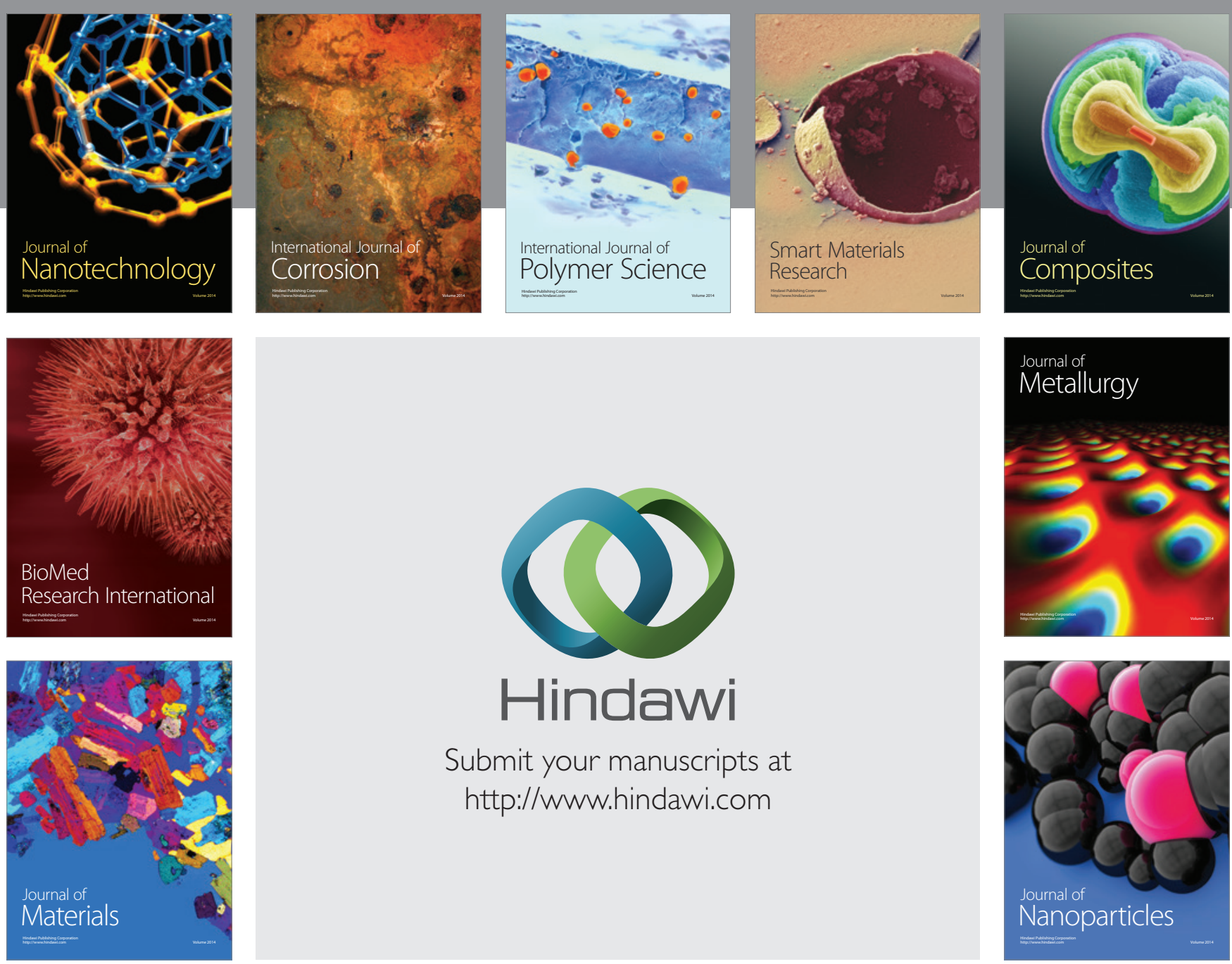

Submit your manuscripts at http://www.hindawi.com
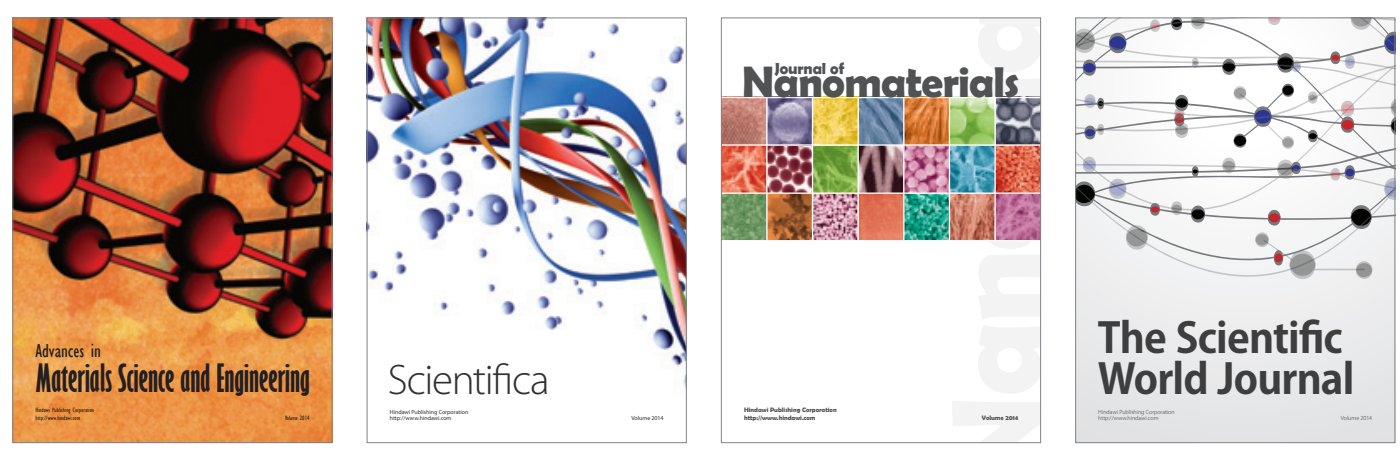

\section{The Scientific World Journal}
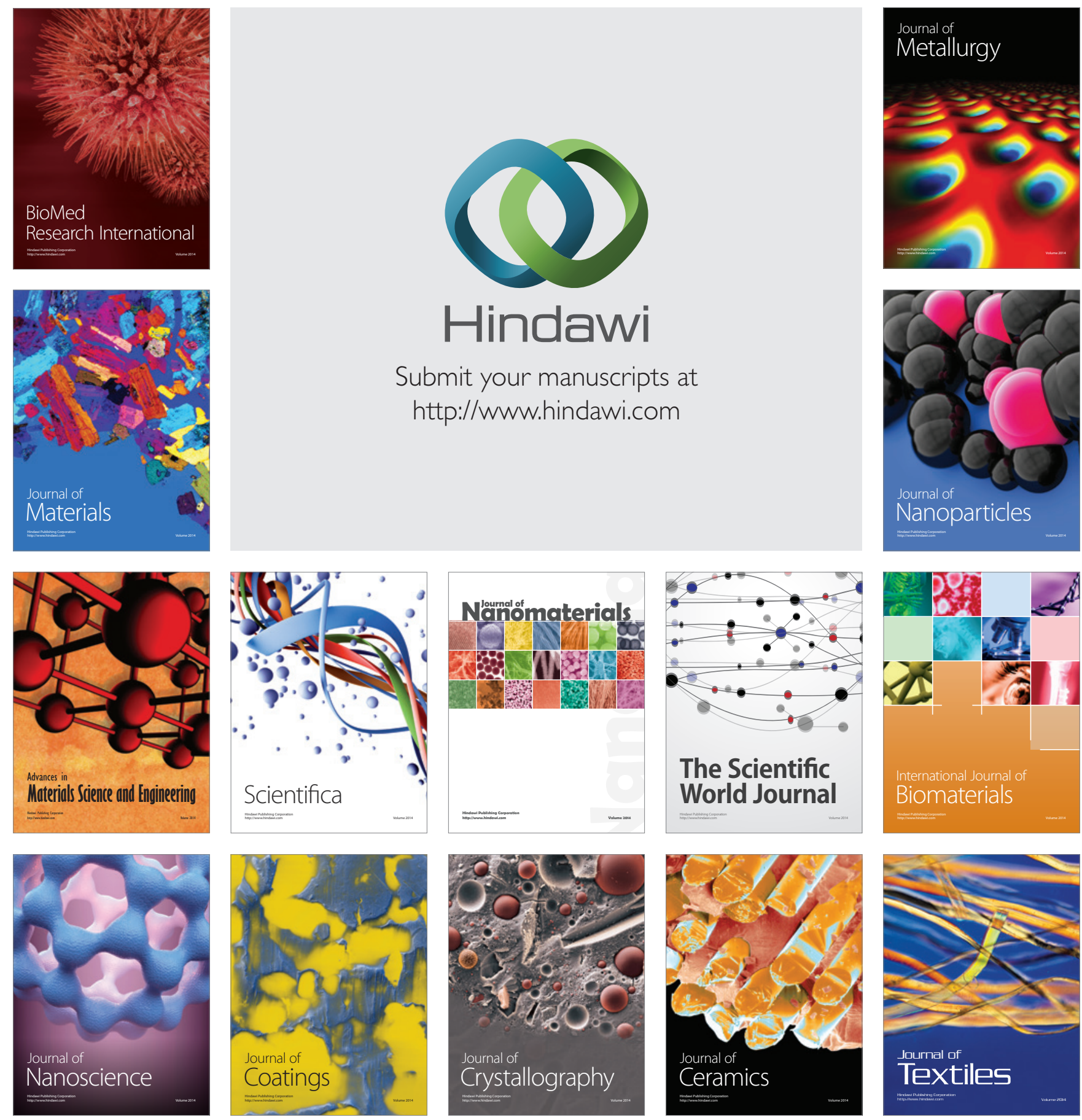